\title{
Can Higher Body Mass Index Patients Save Blood Following On-pump Coronary Artery Bypass Grafting?
}

\author{
Wenyuan Zhang, MD, ${ }^{1}$ Shibiao Chen, $\mathrm{PhD},{ }^{1}$ Xiaojie Liu, MD,${ }^{2}$ Lijuan Wang, $\mathrm{MD},{ }^{2}$ Sudena Wang, MD, ${ }^{2}$ \\ Yang $\mathrm{Yu}, \mathrm{MD},{ }^{2}$ Hushan $\mathrm{Ao}, \mathrm{PhD}^{2}$ \\ ${ }^{1}$ Department of Anesthesiology, The First Affiliated Hospital, Nanchang University, Nanchang, Jiangxi Sheng, China; ${ }^{2}$ Department of \\ Anesthesiology, State Key Laboratory of Cardiovascular Disease, National Clinical Research Center of Cardiovascular Diseases, Fuwai \\ Hospital, for Cardiovascular Diseases, Chinese Academy of Medical Sciences and Peking Union Medical College. No.167 North Lishi \\ Road, Xicheng District, Beijing, China
}

\section{ABSTRACT}

Background: The association of obesity with postoperative bleeding volume and transfusions after coronary artery bypass grafting $(\mathrm{CABG})$ is not clear. We evaluated the effects of body mass index (BMI) on bleeding volume and transfusions in patients undergoing on-pump CABG.

Methods: We investigated 1,050 patients, aged 24 years to 83 years, who underwent isolated, primary CABG with cardiopulmonary bypass (CPB) at Fuwai Hospital, Chinese Academy of Medical Sciences, from September 2017 to July 2018. We recorded preoperative medical and laboratory coagulation parameters, intraoperative data, and postoperative bleeding volume for 24 hours following surgery. Primary endpoint was bleeding volume within 24 hours and transfusions after surgery. We analyzed the correlation between bleeding volume within 24 hours and transfusions after surgery and preoperative data with univariate and multivariate linear regression and logistic regression.

Results: Old age significantly increased postoperative transfusions $(\mathrm{OR}=1.03595 \%$ CI 1.013-1.058, $P=.002)$, whereas higher $\mathrm{BMI}(\mathrm{OR}=0.89795 \%$ CI 0.848-0.949, $P<.001)$ and higher hemoglobin (HGB) $(\mathrm{OR}=0.966$ $95 \%$ CI $0.954-0.978, P<0.001)$ decreased postoperative transfusions. And, BMI independently was correlated with bleeding volume after multivariate regression analysis (B $=-0.257, P<.001)$.

Conclusions: Our findings suggest higher BMI may reduce postoperative bleeding volume and transfusion rate, thus such patients may save blood products during onpump CABG.

\section{INTRODUCTION}

Obesity is becoming a worldwide burden. By 2025, the percentage of obesity is expected to account for $18 \%$ in males

Received May 26, 2019; 2019; accepted fuly 9, 2019.

Correspondence: Hushan Ao, No.167 North Lishi Road, Xicheng District, Beijing, China 100037; 0086-10-68006210; fax: 0086-10-68006210 (e-mail:aohushan@126.com). and more than $21 \%$ in females; severe obesity will be more than $6 \%$ in males and $9 \%$ in females [Collaboration NCDRF 2016]. Obesity is on the rise with mean BMI increasing 0.4$0.5 \mathrm{~kg} \mathrm{~m}-2$ annually worldwide [Finucane 2011]. BMI (BMI = weight height-2) is used to quantify obesity nowadays, but it's not a perfect measure because it fails to consider variations, such as muscle bulk and ethnicity. BMI does, however, correlate with percentage body fat in majority clinical situations [Hunt 2017].

Previous studies have revealed that obesity is a risk factor of diabetes mellitus, hypertension, and coronary artery disease [Simopoulos 1984]. However, there is much controversy regarding increased morbidity in obese patients undergoing CABG. A large sample study showed the risk of death increased with a higher BMI [Zheng 2011; Kuduvalli 2002]. However, increased mortality after CABG also is associated with low BMI as underweight [Mariscalco 2017; Reeves 2003].

Severe postoperative bleeding after cardiac surgery can cause certain complications, such as stroke, acute kidney injury, perioperative myocardial infarction, and mortality [Dyke 2014; Ranucci 2013]. Meanwhile, transfusions can induce acute lung injury, postoperative low cardiac output, early and late mortality, and higher costs together with longer stays in the intensive care unit (ICU) [Murphy 2007; van Straten 2010]. Therefore, fewer blood transfusions and avoiding unnecessary transfusions in cardiac surgery are of great importance.

The relationship between obesity and non-obesity based on BMI, postoperative bleeding volume, and blood transfusion remains unclear. Nolan et al [Nolan 2011] reported that rate and amount of transfusion and total blood loss were reduced in obese individuals and indicated a linear correlation between BMI and postoperative bleeding volume. On the other hand, Gurm et al examined that the risk of postoperative bleeding complications does not differ between obese and non-obese patients [Gurm 2002]. Our previous study illustrated the linear correlation between BMI and postoperative bleeding volume in Chinese patients [Wang 2017]. However, no study confirmed its relationship with transfusions in Chinese as far as we know. So, we further explored whether higher BMI can reduce postoperative bleeding volume and transfusions after CABG. 
Table 1. Baseline Characteristics of the Patients $(N=1050)$

\begin{tabular}{|c|c|}
\hline Age (years) & $60.6 \pm 8.33$ \\
\hline Men (\%) & $810(77.1)$ \\
\hline Family history of $\mathrm{CHD}(\%)$ & $130(12.4)$ \\
\hline Hypertension (\%) & $675(64.3)$ \\
\hline Hyperlipidemia (\%) & $858(81.7)$ \\
\hline Diabetes (\%) & $369(35.1)$ \\
\hline Cerebrovascular events (\%) & $83(7.9)$ \\
\hline Myocardial infarction (\%) & $71(6.8)$ \\
\hline Interventional stent (\%) & $31(3.0)$ \\
\hline Heart failure (\%) & $5(0.6)$ \\
\hline Surgery history (\%) & $208(19.8)$ \\
\hline \multicolumn{2}{|l|}{ Medical therapy } \\
\hline LMWH (\%) & $834(79.4)$ \\
\hline Aspirin (\%) & $280(26.7)$ \\
\hline Ticagrelor (\%) & $50(54.8)$ \\
\hline ACEI (\%) & $197(18.8)$ \\
\hline$\beta$-blocker (\%) & $115(11.0)$ \\
\hline LVEF (\%) & $58.5 \pm 11.5$ \\
\hline BMI (kg m-2) & $25.94 \pm 3.22$ \\
\hline HGB (g L-1) & $135.5 \pm 15.7$ \\
\hline PLT count $\left(\times 10^{9} \mathrm{~L}-1\right)$ & $218.1 \pm 58.6$ \\
\hline PCT (\%) & $0.23 \pm 0.06$ \\
\hline PT (sec) & $13.08 \pm 0.83$ \\
\hline APTT (sec) & $36.91 \pm 6.24$ \\
\hline FIB (g L-1) & $3.54 \pm 0.95$ \\
\hline D-dimers ( $\mu \mathrm{g}$ ml-1) & $0.27(0.22,0.40)$ \\
\hline $\mathrm{FDP}(\mu \mathrm{g} \mathrm{ml}-1)$ & $2.5(2.5,2.5)$ \\
\hline CPB time (min) & $106.0 \pm 31.6$ \\
\hline Aortic clamp time (min) & $73.1 \pm 24.1$ \\
\hline Number of anastomoses & $3.50 \pm 0.8$ \\
\hline Postoperative bleeding ( $\mathrm{mL} 24 \mathrm{~h}-1)$ & $390(300,500)$ \\
\hline RBC transfusion (\%) & $208(19.8)$ \\
\hline
\end{tabular}

CHD: coronary heart disease, LMWH: low molecular weight heparin, ACEI: angiotensin-converting enzyme inhibitors, LVEF: left ventricular ejection fraction, BMI: body mass index, HGB: hemoglobin, PLT: platelet, PCT: plateletcrit, PT: prothrombin time, APTT: activated partial thromboplastin time, FIB: fibrinogen, FDP: fibrinogen degradation product, CPB: cardiopulmonary bypass, RBC: red blood cell.

\section{MATERIALS AND METHODS}

Study population: This was a retrospective study. Data was collected on 1,050 patients who underwent isolated primary on-pump CABG at Fuwai Hospital, from September 2017 to July 2018. Isolated primary on-pump CABG

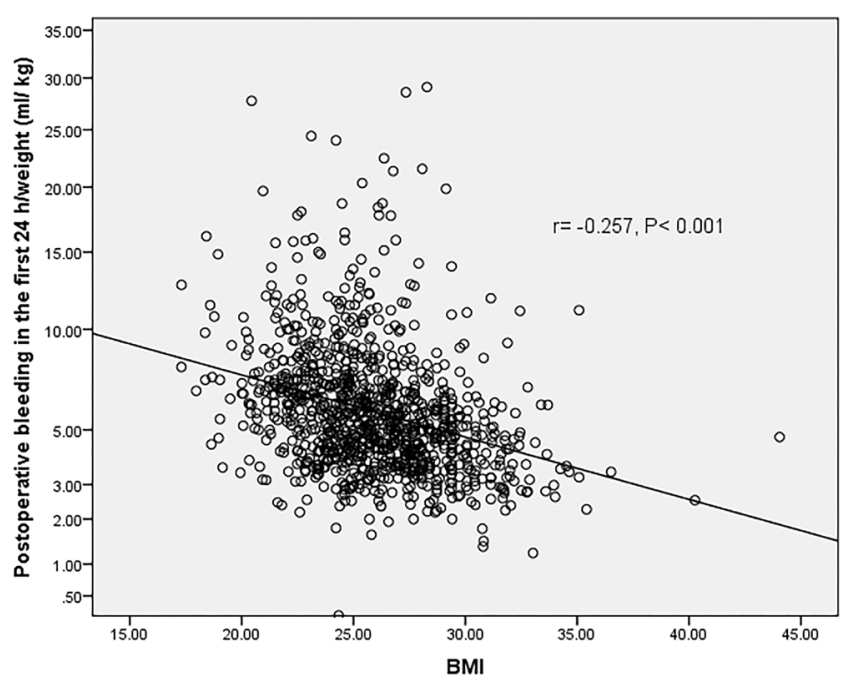

Figure 1. Correlation between BMI and postoperative bleeding. There was a significant correlation between the two factors $(r=-0.257, P<.001)$.

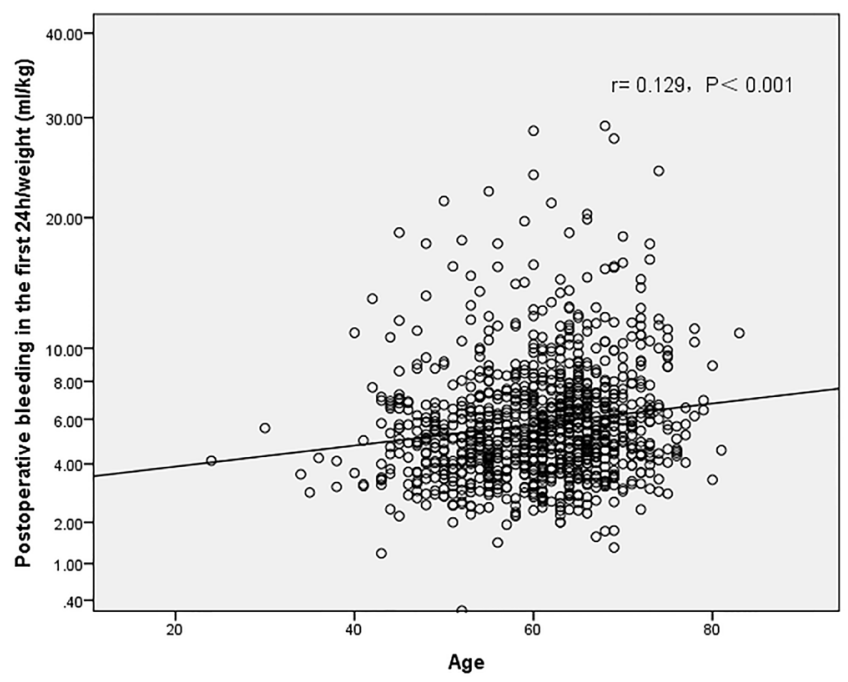

Figure 2. Correlation between age and postoperative bleeding. There was a correlation between the two factors $(r=0.129, P<.001)$.

was defined as coronary artery bypass graft surgery for the first time with cardiopulmonary bypass. The study was approved by the Ethics Committee of Fuwai Hospital, and written informed consent was waived. We excluded patients of emergency CABG, off-pump CABG, concomitant surgical procedures, and patients with liver and kidney dysfunction.

Data collection: We collected preoperative medical therapy, laboratory coagulation parameters [platelet (PLT) count, plateletcrit (PCT), prothrombin time (PT), activated partial thromboplastin time (APTT), D-dimers, fibrinogen (fibrinogen (FIB), and fibrinogen degradation product (FDP)], intraoperative data and postoperative bleeding volume within 24 hours after surgery. 
Table 2. Linear regression analysis for postoperative bleeding volume

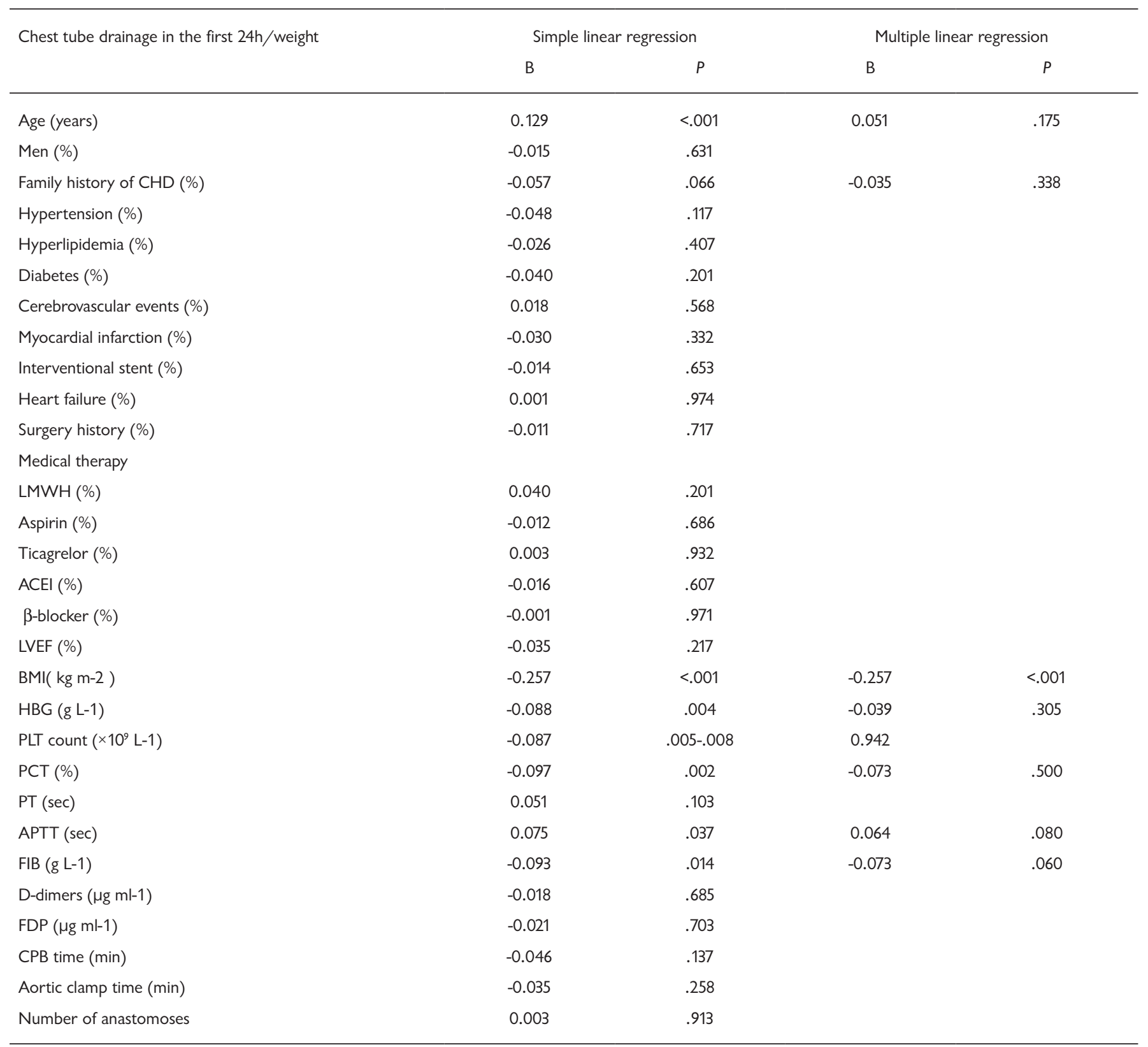

CHD: coronary heart disease, LMWH: low molecular weight heparin, ACEI: angiotensin-converting enzyme inhibitors, LVEF: left ventricular ejection fraction, BMI: body mass index, HGB: hemoglobin, PLT: platelet, PCT: plateletcrit, PT: prothrombin time, APTT: activated partial thromboplastin time, FIB: fibrinogen, FDP: fibrinogen degradation product, CPB: cardiopulmonary bypass

Postoperative bleeding volume was defined as the total amount of chest tube drainage within 24 hours postoperatively, which was recorded by a nurse. We tried to eliminate interference by recording volume of chest tube drainage weight- 1 rather than volume of chest tube drainage to prevent being affected by individual weight.

Statistical analysis: Categorical variables were expressed as numbers and percentage, and continuous variables were expressed in the form of mean \pm standard deviation (for normally distributed continuous variable comparisons between groups) or the median (IQR) (for abnormally distributed continuous variable comparisons between groups).

Simple linear regression and logistic regression were used to analyze the relationship between demographic data and postoperative bleeding volume and transfusion. A multiple linear regression model was used to identify the independent variable associated 
Table 3. Multiple logistic regression analysis for postoperative transfusion

\begin{tabular}{lccc}
\hline Postoperative transfusion & Adjusted OR & $95 \% \mathrm{Cl}$ & $P$ \\
\hline Age (years) & 1.035 & $1.013-1.058$ & .002 \\
Men (\%) & 0.910 & $0.611-1.355$ & .643 \\
BMl (kg m-2) & 0.897 & $0.848-0.949$ & $<.001$ \\
HGB (g L-1) & 0.966 & $0.954-0.978$ & $<.001$ \\
PLT count (×109 L-1) & 0.997 & $0.989-1.005$ & .437 \\
PCT (\%) & 2.102 & $0.001-1.101$ & .865 \\
\hline
\end{tabular}

BMI: body mass index, HGB: hemoglobin, PLT: platelet, PCT: plateletcrit

with postoperative bleeding volume, and multiple logistic regression analysis was used to identify the independent variable associated with postoperative transfusion. For both models, covariates were selected into the multiple linear regression models if the $P$ value of any independent variable was less than 0.1 after univariate regression analysis.

All statistical analyses were performed with SPSS version 23.0 software (SPSS Inc., Chicago, IL, USA).

\section{RESULTS}

Demographic and clinical data of the patients are presented in Table 1. It is obvious that postoperative bleeding volume significantly correlated with BMI $(P<.001)$ (Figure 1$)$, age $(P<.001)$ (Figure 2$), \mathrm{HGB}(P<.01)$ (Figure 3$)$, PLT count $(P<.01)$, PCT $(\mathrm{P}<0.01)$, APTT $(\mathrm{P}<0.05)$, and FIB $(P<.05)$. However, BMI was an independent predictor of postoperative bleeding volume by the multiple linear regression $(P<.001)$ (Table 2$)$.

Old age significantly increased the rate of postoperative transfusions $(\mathrm{OR}=1.03595 \%$ CI 1.013-1.058, $P=.002)$, whereas higher $\mathrm{BMI}(\mathrm{OR}=0.89795 \%$ CI $0.848-0.949$, $P<.001)$ and higher HBG (OR $=0.96695 \%$ CI $0.954-0.978$, $P<.001$ ) decreased postoperative transfusions (Table 3 ).

\section{DISCUSSION}

Our study verified the correlation between BMI and postoperative bleeding volume and transfusion. We draw the conclusion that BMI, even within a normal range, can inhibit postoperative bleeding volume and a reduced transfusion rate can be considered as a biomarker to evaluate patients undergoing cardiac surgery.

This study demonstrated BMI is an independent preoperative indicator of postoperative bleeding volume. Although, some factors can contribute to postoperative bleeding volume, including amount of fluid left in the pleural cavity from intraoperative blood loss, for example. But there is evidence that chest-tube location does not affect

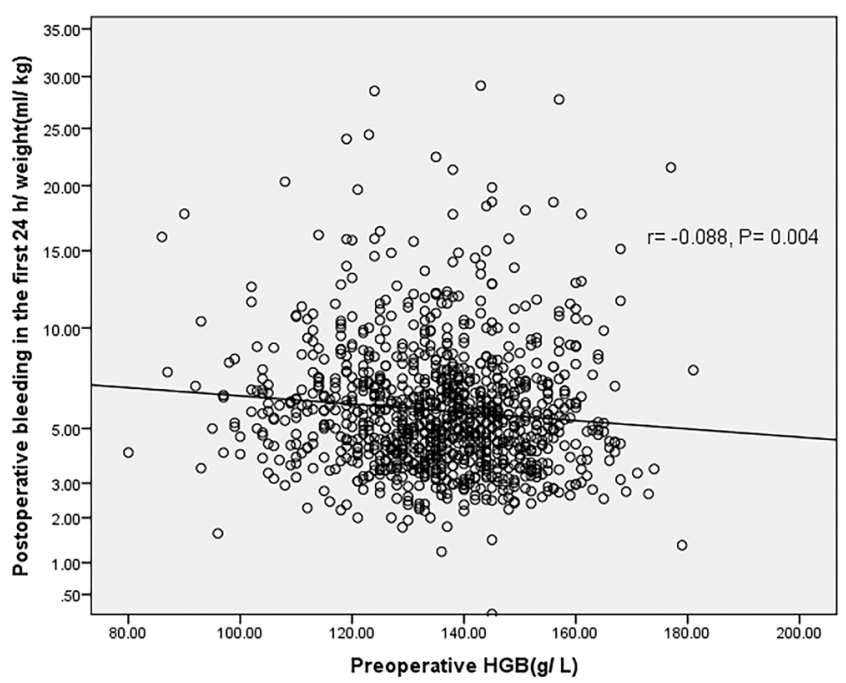

Figure 3. Correlation between HGB and postoperative bleeding. There was a correlation between the two factors $(r=-0.088, P=.004)$.

output [Sensoz 2011]. Chest tube drainage, therefore, is considered to be the only objective indicator of postoperative bleeding volume. As mentioned above, several studies [Gurm 2002; Nolan 2013] evaluated the correlation of BMI and postoperative bleeding volume and transfusions, which were similar with our findings that decreased postoperative bleeding volume and transfusions occurred in an obese group. However, the mechanisms were unexplored. Besides, there are some drawbacks of this grouping method, such as the BMI varying widely in each group, which may not reflect the effects of continuous BMI on postoperative bleeding volume and transfusions.

The link between BMI and postoperative bleeding volume and transfusions may relate to the procoagulant state. Obese patients have higher plasma concentrations of coagulation factor II (FVII), levels of thrombin and thrombinantithrombin (TAT) complexes, and procoagulant activity of tissue factor (TF) [Samad 2013]. On the other hand, weight loss in morbidly obese patients significantly reduces thrombin generation potential and decreases levels of circulating TF, FVII [Ay 2010; Kopp 2012]. Obesity also is correlated with increased platelet activation [Santilli 2012].

Age previously has shown to be an independent risk factor for blood transfusion after cardiac surgery [Frankel 2005], which is in accordance with the results of our study.

Even though we tried our best, there are still several limitations. First, this study was conducted in a single center of ethnic Chinese patients which may not reflect other patients worldwide. Second, as a retrospective study, we could only collect postoperative data, thus, perioperative fluid management was not assessed.

In summary, this study illustrated blood loss and transfusions correlated with BMI. BMI may be used as an indicator to predict postoperative bleeding volume and transfusions. Therefore, we strongly recommended taking BMI into account in preoperative assessment, which will provide a more accurate preoperative prediction of CABG outcome. 


\section{REFERENCES}

Ay L, Kopp HP, Brix JM. 2010. Thrombin generation in morbid obesity: significant reduction after weight loss. J Thromb Haemost 8: 759-765.

Collaboration NCDRF. 2016. Trends in adult body-mass index in 200 countries from 1975 to 2014: a pooled analysis of 1698 population-based measurement studies with 19.2 million participants. Lancet 387: 1377-96.

Dyke C, Aronson S, Dietrich W, et al. 2014. Universal definition of perioperative bleeding in adult cardiac surgery. J Thorac Cardiovasc Surg 147: $1458-1463$.

Finucane MM, Stevens GA, Cowan MJ, et al. 2011. National, regional, and global trends in body-mass index since 1980: systematic analysis of health examination surveys and epidemiological studies with 960 country years and 9.1 million participants. Lancet 377: 557-67.

Frankel TL, Stamou SC, Lowery RC, et al. 2005. Risk factors for hemorrhagerelated reexploration and blood transfusion after conventional versus coronary revascularization without cardiopulmonary bypass. Eur J Cardiothorac Surg 27: 494-500.

Gurm HS, Whitlow PL, Kip KE. 2002. The impact of body mass index on short- and long-term outcomes in patients undergoing coronary revascularization. Insights from the bypass angioplasty revascularization investigation (BARI). J Am Coll Cardiol 39: 834-40.

Hunt BJ. 2017. The effect of BMI on haemostasis: Implications for thrombosis in women's health. Thrombosis research 151: S53-S5.

Kopp CW, Kopp HP, Steiner S. 2003. Weight loss reduces tissue factor in morbidly obese patients. Obes Res 11: 950-956.

Kuduvalli M, Grayson AD, Oo AY, Fabri BM, Rashid A. 2002. Risk of morbidity and in-hospital mortality in obese patients undergoing coronary artery bypass surgery. Eur J Cardiothorac Surg 22: 787-793.

Mariscalco G, Wozniak MJ, Dawson AG, et al. 2017. Body Mass Index and mortality among adults undergoing cardiac surgery: a nationwide study with a systematic review and meta-analysis. circulation 135: 850-63.

Murphy GJ, Reeves BC, Rogers CA, et al. 2007. Increased mortality, postoperative morbidity, and cost after red blood cell transfusion in patients having cardiac surgery. Circulation 116: 2544-52.
Nolan HR, Davenport DL, Ramaiah C. 2013. BMI is an independent preoperative predictor of intraoperative transfusion and postoperative chest-Tube output. The Int J Angiol 22: 31-6.

Nolan HR, Ramaiah C. 2011. Effect of body mass index on postoperative transfusions and 24-hour chest-tube output. The International journal of angiology 20: 81-6.

Ranucci M, Baryshnikova E, Castelvecchio S, Pelissero G, Surgical and Clinical Outcome Research (SCORE) Group. 2013. Major bleeding, transfusions, and anemia: the deadly triad of cardiac surgery. Ann Thorac Surg 96: 478-85.

Reeves BC, Ascione R, Chamberlain MH, Angelini GD. 2003. Effect of body mass index on early outcomes in patients undergoing coronary artery bypass surgery. J Am Coll Cardiol 42:668-676.

Samad F, Ruf W. 2013. Inflammation, obesity, and thrombosis. Blood 122: 3415-22.

Santilli F, Vazzana N, Liani R, Guagnano MT, Davì G. 2012. Platelet activation in obesity and metabolic syndrome. Obes Rev 13: 27-42.

Sensoz Y, Gunay R, Tuygun AK, et al. 2011. Computed tomography evaluation of different chest tube sites for residual pleural volumes after coronary artery bypass surgery. Ann Saudi Med 31: 383-386.

Simopoulos AP, Van Itallie TB. 1984. Body weight, health, and longevity. Ann Intern Med 100: 285-95.

van Straten AH, Bekker MW, Soliman Hamad MA, et al. 2010. Transfusion of red blood cells: the impact on short-term and long-term survival after coronary artery bypass grafting, a ten-year follow-up. Interactive cardiovascular and thoracic surgery 10: 37-42.

Wang M, Chen M, Ao H, Chen S, Wang Z. 2017. The Effects of Different BMI on Blood Loss and Transfusions in Chinese Patients Undergoing Coronary Artery Bypass Grafting. Annals of thoracic and cardiovascular surgery. official journal of the Association of Thoracic and Cardiovascular Surgeons of Asia 23: 83-90.

Zheng W, McLerran DF, Rolland B, et al. 2011. Association between body-mass index and risk of death in more than 1 million Asians. The New England journal of medicine. 364: 719-29. 